Tropical Journal of Pharmaceutical Research May 2019; 18 (5): 1101-1108

ISSN: $1596-5996$ (print); 1596-9827 (electronic)

(C) Pharmacotherapy Group, Faculty of Pharmacy, University of Benin, Benin City, 300001 Nigeria.

\title{
Synthesis, antimicrobial activities and GAPDH docking of novel 1, 2, 3-triazole derivatives
}

\author{
Abdulsalam AM Alkhaldi ${ }^{1}$, Mohamed A Abdelgawad ${ }^{2,3^{*},}$ Bahaa GM Youssif $^{2,4}$, \\ Ahmed O El-Gendy ${ }^{5}$, Harry P De Koning ${ }^{6}$ \\ ${ }^{1}$ Biology Department, College of Science, Jouf University, Sakaka, Aljouf 2014, Saudi Arabia, ${ }^{2}$ Department of Pharmaceutical \\ Chemistry, College of Pharmacy, Jouf University, Sakaka, Aljouf 2014, Saudi Arabia, ${ }^{3}$ Department of Pharmaceutical Organic \\ Chemistry, Faculty of Pharmacy, Beni-Suef University, Beni-Suef 62514, Egypt, ${ }^{4}$ Department of Pharmaceutical Organic \\ Chemistry, Faculty of Pharmacy, Assiut University, Assiut 71526, Egypt, ${ }^{5}$ Department of Microbiology \& Immunology, Faculty \\ of Pharmacy, Beni-Suef University, Beni-Suef 62514, Egypt, ${ }^{6}$ Institute of Infection, Immunity and Inflammation, College of \\ Medical, Veterinary and Life Sciences, University of Glasgow, Glasgow G12 8TA, United Kingdom
}

${ }^{*}$ For correspondence: Email: mohamedabdelwahab976@yahoo.com; Tel: 0020238482600

Sent for review: 1 February 2019

Revised accepted: 24 April 2019

\begin{abstract}
Purpose: To synthesize new triazole derivatives in order to overcome the problem of side effects of antimicrobial agents and microbial resistance, while broadening the spectrum of antimicrobial activity. Methods: The starting triazole, compound 1, was prepared through click chemistry and reacted with chloroacetyl chloride to yield compound II. Triazole 1 was reacted with acids and aldehydes to produce oxadiazole (III) and azomethine (IV) which cyclized in acetic anhydride to give a new acetylated oxadiazole (V). Minimum inhibitory concentration (MIC) and resorufin assays were used for antibacterial and anti-parasitic screening, respectively. Compounds II and IVb were subjected to molecular docking studies using glyceraldehyde-3-phosphate dehydrogenase (GAPDH) Molecular Operating Environment (MOE) program.

Results: Novel oxazole-triazole derivative (III) showed high activity against Pseudomonas aeruginosa and moderate activity against Staphylococcus epidermidis, whereas compound IVc showed moderate activity against Staphylococcus epidermidis. Chloro-acetyl-triazole II and 2-hydroxyphenyl-triazole Schiff base (Ivb) showed pronounced activity against the kinetoplastid parasites, Leishmania major, Leishmania mexicana and Trypanosoma brucei.

Conclusion: The new synthesized triazoles represent a new antimicrobial scaffold and identifies potential new lead compounds for follow-up and for further mechanistic studies.
\end{abstract}

Keywords: Antimicrobial, Triazole, Trypanosoma, Leishmania, Kinetoplastid

This is an Open Access article that uses a fund-ing model which does not charge readers or their institutions for access and distributed under the terms of the Creative Commons Attribution License (http://creativecommons.org/licenses/by/4.0) and the Budapest Open Access Initiative (http://www.budapestopenaccessinitiative.org/read), which permit unrestricted use, distribution, and reproduction in any medium, provided the original work is properly credited.

Tropical Journal of Pharmaceutical Research is indexed by Science Citation Index (SciSearch), Scopus, International Pharmaceutical Abstract, Chemical Abstracts, Embase, Index Copernicus, EBSCO, African Index Medicus, JournalSeek, Journal Citation Reports/Science Edition, Directory of Open Access Journals (DOAJ), African Journal Online, Bioline International, Open-J-Gate and Pharmacy Abstracts

\section{INTRODUCTION}

Compounds containing triazole cores have numerous pharmacological activities and applications in the pharmaceutical field [1 - 4] . Combination of a triazole with triazines (3), pyridines (2), pyridazines (3), pyrazines (1), and pyrimidines (4) form new scaffolds with 
pronounced activities. The incorporation of the $\mathrm{N}-\mathrm{C}-\mathrm{S}$ skeleton into triazole and thiadiazine produces compounds with significant antimicrobial activities [5]. The nucleophilic amino group is the center for the synthesis of heterocyclic rings [6] and for obtaining known Schiff base antimicrobial derivatives [7]. On the other hand, derivatives of oxadiazole are reported to display significant antimicrobial activities, and the merging of two or more different pharmacophores is considered a promising avenue for the design of potent antimicrobials against which resistance does not easily develop [8].

The main kinetoplastid pathogens are classified in the genera Trypanosoma and Leishmania. Leishmaniasis is the result of a Leishmania infection, whereas an infection of Trypanosoma brucei rhodesiense or Trypanosoma brucei gambiense results in sleeping sickness (human African trypanosomiasis, HAT). Moreover, the parasite Trypanosoma cruzi is responsible for Chagas disease, an often-fatal infection particularly prevalent in South and Central America. Worldwide, some 20 million people are infected with these kinetoplastid pathogens, resulting in estimated 95,000 deaths per year [9]. The main challenges of kinetoplastid disease therapies are extensive toxicity, lack of efficacy and potential resistance of the Leishmania species [10], T. brucei [11] and T. cruzi [12]. In the present study, the main target was the synthesis and evaluation of antimicrobial and anti-kinetoplastid parasite activities in new scaffolds of phenyl-triazole rings merged with azomethine, oxadiazole and acetylated oxadiazole rings which are expected to confer high antimicrobial activities with reduced toxicities. The target compounds were obtained through the synthetic pathways shown in Scheme 1.

\section{EXPERIMENTAL}

\section{Chemistry}

The identities, purity and structures of the novel compounds were confirmed according to reported methods [13-15] using the following techniques (equipment in parenthesis): melting point (uncorrected, Thomas-Hoover Capillary Apparatus), IR (Magna FT-IR spectrometer), mass spectroscopy (Hewlett Packard 5988), NMR spectroscopy (400 MHz NMR Bruker device), TLC (Sigma Aldrich Company), and elemental analysis. Compounds I and IVa were prepared as reported earlier [16]. All chemicals were supplied from Sigma Aldrich Company.

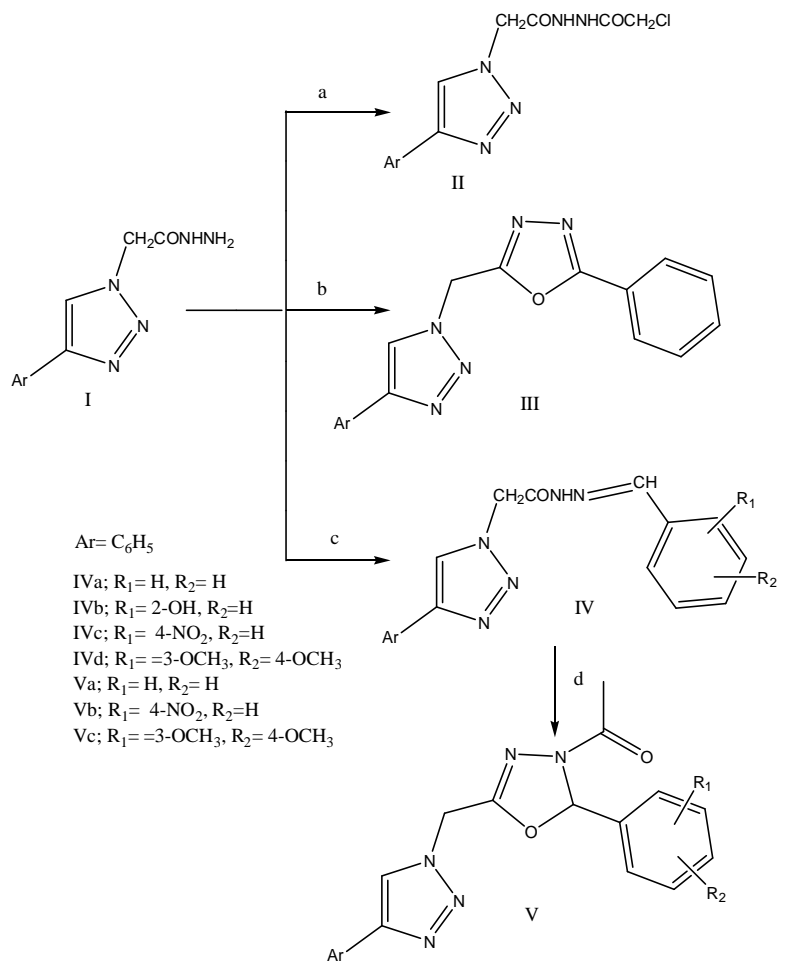

Scheme 1: The synthetic pathway for target compounds

Reagent and reaction conditions: (a) chloroacetyl chloride, anhydrous $\mathrm{Na}_{2} \mathrm{CO}_{3}$, dry $\mathrm{DMF} ;$ b) $\mathrm{POCl}_{3}$, benzoic acid, reflux; c) aromatic aldehyde, ethanol, reflux; d) acetic anhydride, reflux

Chloroacetic acid $N$-[2-(4-phenyl-[1, 2, 3] triazol-1-yl) acetyl] hydrazide (II)

The hydrazide compound I (0.01 mole) was dissolved in dry DMF $(20 \mathrm{~mL})$ containing anhydrous sodium carbonate $\left(\begin{array}{ll}1 & \mathrm{~g}\end{array}\right)$. Chloroacetyl chloride $(0.015$ moles) was added dropwise to stirred solution of the hydrazide. The reaction solution was continually stirred for $4 \mathrm{~h}$, and was thereafter neutralized with sodium carbonate solution (10\%) until alkaline to litmus paper. The product was filtered, washed with ice-cooled water, dried and crystallized from ethanol.

\section{2-Phenyl-5-(4-phenyl-[1, 2, 3]-triazol-1- ylmethyl) $[1,3,4]$ oxadiazole (111)}

The mixture of hydrazide compound I (0.01 mole) and benzoic acid ( 0.01 mole) was added to phosphorus oxychloride $(30 \mathrm{~mL})$. The reaction mixture was refluxed for $8 \mathrm{~h}$ after which it was cooled in an ice bath and added to crushed ice $(100 \mathrm{~g})$. The reaction solution was then neutralized with sodium carbonate solution $(10 \%)$ until it became basic to litmus paper. 
The precipitated product was filtered and washed with cold water. Then, compound III was crystallized from ethanol.

\section{Preparation of compounds IVa - IVd}

Appropriate amounts of aldehyde (0.01 mole) and hydrazide compound I (0.01 mole) in ethanol $(50 \mathrm{~mL})$ with two drops of glacial acetic acid, were refluxed for $10 \mathrm{~h}$. The reaction solution was cooled in ice bath, filtered, and crystallized from ethanol.

\section{Synthesis of compounds Va - Vc}

Azomethine compound IV ( 0.01 mole) and acetic anhydride $(30 \mathrm{~mL})$ were refluxed for $10 \mathrm{~h}$. The reaction solution was cooled in ice bath and continuously stirred with distilled water $(100 \mathrm{~mL})$ for $6 \mathrm{~h}$. Thereafter, the new oxadiazoles were filtered and crystallized from ethanol.

\section{Assessment of antibacterial activity}

The microorganisms used were ATCC or LMG standard isolates kindly provided by Beni-Suef University, Faculty of Pharmacy Microbiology and Immunology Department. The minimum inhibitory concentrations (MICs) of the synthesized compounds were determined using the agar dilution method according to the Clinical Laboratory Standards Institute (CLSI) [17]. For each sample, different concentrations were diluted with Muller Hinton agar to give final concentrations ranging from 200 to $6.25 \mu \mathrm{g} / \mathrm{mL}$. Dimethyl sulphoxide (DMSO) was used as a negative control plate. All bacterial isolates were sub-cultured on Brain Heart Infusion Agar (BHIA) and incubated at $37{ }^{\circ} \mathrm{C}$ for $24 \mathrm{~h}$ [17]. Each experiment was performed in duplicate.

\section{Determination of anti-kinetoplastid activity}

All parasite strains were kept and cultured at the University of Glasgow, and were standard strains used in many published papers [28].

\section{Culturing of Trypanosoma brucei blood- stream forms (BSF) in-vitro}

Two strains of the bloodstream forms of Trypanosoma brucei were utilized. The first strain was the wild type of Trypanosoma brucei (s427WT) and the other was clone B48, which was acquired from a TbAT1-KO strain created by the genetic deletion of the TbAT1 gene from s427WT, followed by in vitro exposure to incremental concentrations of pentamidine, creating a high level of resistance to pentamidine, diminazene and melaminophenyl arsenicals [19]. The culturing of Trypanosoma brucei bloodstream forms (BSF) in vitro was done according to the method reported earlier [20].

\section{Culturing of Leishmania major and Leishmania mexicana promastigotes}

Leishmania major strain Friedlin (LmjF) and a strain of Leishmania mexicana (MNYC/BZ/62/ M379) were propagated in essential medium (HOMEM) at a pH of 7.4 and were supplemented with a $10 \%$ heat-inactivation FCS at $25^{\circ} \mathrm{C}$, as described earlier [21]. The resultant cultures were then passed through fresh medium three times per week.

\section{Resorufin assay}

Resorufin sodium salt (Alamar blue) is usually used as an indicator of cell metabolic functions. It is a non-fluorescent blue dye that is metabolized by live cells to a red fluorescent compound resorufin. The assay was done according to the method reported in [22].

\section{Alamar blue assay}

This assay was essentially performed as described previously [23]. Drug solutions (20 $\mathrm{mM}$ ) for testing in the resazurin assay were prepared in DMSO, ensuring a final concentration of exactly $0.5 \%$ DMSO in the cell suspension (this level of DMSO does not influence cell growth). Plates (96-well) were set up with test concentrations starting from $100 \mu \mathrm{M}$ and 23 double dilutions over two rows of the plate, with the final well used as a drug-free control. Each well of the plate received $100 \mu \mathrm{L}$ of medium; the first well in the dilution series also received $100 \mu \mathrm{L}$ of the $200 \mu \mathrm{M}$ test compound; $100 \mu \mathrm{L}$ went to the next well and so on, until 23 dilutions were made. To each well was then added $100 \mu \mathrm{L}$ of cell suspension containing $2 \times$ $10^{5}$ cells $/ \mathrm{mL}$, and the plates were incubated for $48 \mathrm{~h}$ at $37 \stackrel{\circ}{\circ} \mathrm{C}$ in an incubator containing $5 \% \mathrm{CO}_{2}$, after which $20 \mu \mathrm{L}$ of the resazurin solution was added to each well, followed by a further $24 \mathrm{~h}$ incubation. Fluorescence was then read using a FLUOstar Optima fluorimeter (BMG Labtech; $\lambda_{\text {excitation }}=530 \mathrm{~nm}$ and $\left.\lambda_{\text {emission }}=590 \mathrm{~nm}\right)$. The 50 $\%$ effective concentrations $\left(E_{50}\right)$ were calculated from a plot of the data using the equation for a sigmoidal curve with a variable slope, with Prism 5.0 software (GraphPad Software Inc., California, USA).

\section{Resazurin assay}

The resazurin assay for $L$. major promastigotes and L. mexicana was performed exactly as 
described earlier [24]. It was very similar to the procedure for Trypanosoma brucei, except for the use of HOMEM medium (Section 3.3.2) and $1 \times 10^{5}$ cells per well. The incubation time was $72 \mathrm{~h}$ prior to the addition of the resazurin solution, with additional incubation for $24 \mathrm{~h}$ after the addition.

\section{Molecular modelling studies}

In this study, Molecular Operating Environment (MOE) version 2010.08 (Chemical Computing Group Inc., Montreal, Quebec, Canada) was used in the docking experiments. The crystal structure of nicotinamide adenine dinucleotide (NAD) bound to glyceralde-3-phosphate dehydrogenase (GADPH, PDB: ID 1GYP) was obtained from the RCSB Protein Data Bank. Docking of the co-crystallized ligands was used to study the scoring energies, root mean values, and interactions of amino acids [18].

\section{RESULTS}

\section{Spectra data}

Chloroacetic acid N-[2-(4-phenyl-[1, 2, 3] triazol-1-yl) acetyl] hydrazide (II). White powder (90\%); mp 180 - $184{ }^{\circ} \mathrm{C}$; IR (film) 3109 $(\mathrm{NH}), 3074(\mathrm{CH}$, aromatic), $2960(\mathrm{CH}$, aliphatic), 1666 (broad, $2 \mathrm{C}=\mathrm{O}$ ) $\mathrm{cm}^{-1}$; ${ }^{1} \mathrm{HNMR}\left(\mathrm{DMSO}-d_{6}\right) \delta$ 4.46(s, 2H, CH$\left.{ }_{2} \mathrm{Cl}\right), 5.60\left(\mathrm{~s}, 2 \mathrm{H}, \mathrm{CH}_{2}\right), 7.34-$ $7.38(\mathrm{~m}, 1 \mathrm{H}$, phenyl $\mathrm{H}-4), 7.45-7.48(\mathrm{~m}, 2 \mathrm{H}$, phenyl $\mathrm{H}-3,5), 7.88-7.89$ (m, $2 \mathrm{H}$, phenyl $\mathrm{H}-2,6)$, $8.71(\mathrm{~s}, 1 \mathrm{H}$, triazole $\mathrm{H}), \mathrm{NH}$ peaks not observable; ${ }^{13} \mathrm{C}$ NMR (DMSO- $d_{6}$ ) $\delta$ 44.47, $50.75,122.69,123.43,125.62,129.44,130.76$, 146.69, 152.86, 162.05; EIMS (m/z) $293\left(\mathrm{M}^{+}\right.$, $12.79 \%), \quad 45$ (100\%); Anal. Calcd for $\mathrm{C}_{12} \mathrm{H}_{12} \mathrm{CIN}_{5} \mathrm{O}_{2}$ : C, 49.07; $\mathrm{H}, 4.12 ; \mathrm{N}, 23.84$; found: C, 49.10; H, 4.10; N, 23.80.

2-Phenyl-5-(4-phenyl-[1, 2, 3]-triazol-1ylmethyl) $[1,3$, 4]oxadiazole (111). Dirty white powder (60\%); mp 258-260 ${ }^{\circ} \mathrm{C}$; IR (film) $3039(\mathrm{CH}$, aromatic), $2900(\mathrm{CH}$, aliphatic), 1600 $(\mathrm{C}=\mathrm{N}) \mathrm{cm}^{-1} ;{ }^{1} \mathrm{H}$ NMR (DMSO- $\left.d_{6}\right) \delta 5.30,5.32$ $\left(\mathrm{s}, 2 \mathrm{H}, \mathrm{CH}_{2}\right), 7.36-7.47(\mathrm{~m}, 6 \mathrm{H}$, two phenyl $\mathrm{H}$ 3,4,5), 7.86-7.88 (m, 4H, two phenyl $\mathrm{H}-2,6)$, $8.56(\mathrm{~s}, 1 \mathrm{H}$, triazole $\mathrm{H})$; Anal. Calcd for $\mathrm{C}_{17} \mathrm{H}_{13} \mathrm{~N}_{5} \mathrm{O}$ : C, 67.32; $\mathrm{H}, 4.32 ; \mathrm{N}, 23.09$; found: C, 67.50; H, 4.20; N, 23.00 .

(4-Phenyl-[1,2,3]-triazol-1-yl) acetic acid (2hydroxybenzylidene) hydrazide (IVb). White powder (70\%); mp $233-235{ }^{\circ} \mathrm{C}$; IR (film) 3487 $(\mathrm{OH}), 3444(\mathrm{NH}), 1697(\mathrm{C}=\mathrm{O}), 1616(\mathrm{C}=\mathrm{N}) \mathrm{cm}^{-}$ ${ }^{7}{ }^{1} \mathrm{H}$ NMR (DMSO-d $\left.d_{6}\right) \delta 5.33,5.74\left(\mathrm{~s}, 2 \mathrm{H}, \mathrm{CH}_{2}\right)$, 6.86-6.93 ( $\mathrm{m}, 2 \mathrm{H}$, hydroxylphenyl- $\mathrm{H}-3, \mathrm{H}-5)$, 7.25-7.37 $(\mathrm{m}, 2 \mathrm{H}$, hydroxyphenyl $\mathrm{H}-4$, phenyl $\mathrm{H}$ -
4), 7.45-7.49 (m, $2 \mathrm{H}$, phenyl $\mathrm{H}-3,5)$, 7.807.81( $\mathrm{m}, 1 \mathrm{H}$, hydoxyphenyl $\mathrm{H}-6), 7.87-7.90(\mathrm{~m}$, $2 \mathrm{H}$, phenyl $\mathrm{H}-2,6), 8.30(\mathrm{~s}, 1 \mathrm{H}$, triazole), $8.56(\mathrm{~s}$, $\mathrm{H}, \mathrm{N}=\mathrm{CH}), 10.2(\mathrm{~s}, 1 \mathrm{H}, \mathrm{NH}), 11.82(\mathrm{~s}, 1 \mathrm{H}, \mathrm{OH}$, $\mathrm{D}_{2} \mathrm{O}$ exchangeable); ${ }^{13} \mathrm{C}$ NMR (DMSO- $d_{6}$ ) $\delta$ 51.47, 116.63, 119.91, 120.46, 123.46, 125.59, $126.41,128.40,131.11,132.19, \quad 142.26$, 146.78. 148.33, 156.94, 167.47; Anal. Calcd for $\mathrm{C}_{17} \mathrm{H}_{15} \mathrm{~N}_{5} \mathrm{O}_{2}: \mathrm{C}, 63.54 ; \mathrm{H}, 4.71 ; \mathrm{N}, 21.79$; found: $\mathrm{C}, 63.50 ; \mathrm{H}, 4.60 ; \mathrm{N}, 21.90$.

(4-Phenyl-[1, 2, 3]-triazol-1-yl) acetic acid (4nitrobenzylidene) hydrazide (IVc). Yellowish white powder (90\%); mp 226-228 ${ }^{\circ} \mathrm{C}$; IR (film) $3452(\mathrm{NH}), 1697(\mathrm{C}=\mathrm{O}), 1627(\mathrm{C}=\mathrm{N}) \mathrm{cm}-1 ;{ }^{1} \mathrm{H}$ NMR (DMSO-d6) $\delta$ 5.30-5.82 (m, 2H, $\mathrm{CH}_{2}$ ), 7.34$7.35(\mathrm{~m}, 2 \mathrm{H}$, phenyl $\mathrm{H}-2,6), 7.44-7.48(\mathrm{~m}, 3 \mathrm{H}$, phenyl $\mathrm{H}-3,4,5)$, 7.85-7.89 $(\mathrm{m}, 3 \mathrm{H}$, triazole- $\mathrm{H}$, 4nitrophenyl $\mathrm{H}-2,6)$, 8.02-8.37 ( $\mathrm{m}, 2 \mathrm{H}$, nitrophenyl $\mathrm{H}-3,5), 8.51-8.61(\mathrm{~m}, 2 \mathrm{H}, \mathrm{NH}, \mathrm{N}=\mathrm{CH}) ;{ }^{13} \mathrm{C} \mathrm{NMR}$ (DMSO-d6) $\delta$ 51.36, 123.70, 124.51, 125.59, 128.50, 129.40, 131.07, 131.18, 140.64, 142.70, 153.05, 157.54, 167.84. Anal. Calcd for $\mathrm{C}_{17} \mathrm{H}_{14} \mathrm{~N}_{6} \mathrm{O}_{3}$ : C, 58.28; $\mathrm{H}, 4.03$; N, 23.99; found: $\mathrm{C}, 58.10 ; \mathrm{H}, 4.10 ; \mathrm{N}, 24.00$.

(4-Phenyl-[1, 2, 3] triazol-1-yl) acetic acid (3, 4-dimethoxybenzylidene) hydrazide (IVd). White powder (75\%); mp 203-205 ${ }^{\circ} \mathrm{C}$; IR (film) $3262(\mathrm{NH}), \quad 3001(\mathrm{CH}$, aromatic), $2927(\mathrm{CH}$, aliphatic), $1697(\mathrm{C}=\mathrm{O}) \mathrm{cm}-1 ;{ }^{1} \mathrm{H}$ NMR (DMSO-d6) $\delta 3.44\left(\mathrm{~s}, 3 \mathrm{H}, 4-\mathrm{OCH}_{3}\right), 3.47\left(\mathrm{~s}, 3 \mathrm{H}, 3 \mathrm{OCH}_{3}\right)$, 5.57(s, 2H, $\left.\quad \mathrm{CH}_{2}\right), \quad 7.32-7.36 \quad(\mathrm{~m}, \quad 2 \mathrm{H}$, dimethoxyphenyl- $\mathrm{H}-2,5), \quad 7.44-7.48(\mathrm{~m}, \quad 4 \mathrm{H}$, dimethoxyphenyl $\mathrm{H}-6$, phenyl $\mathrm{H}-3,4,5)$, 7.86$7.88(\mathrm{~m}, 3 \mathrm{H}$, phenyl $\mathrm{H}-2,6$, triazole $\mathrm{H}), 8.54(\mathrm{~s}, \mathrm{H}$, $\mathrm{N}=\mathrm{CH}), 10.63(\mathrm{~s}, 1 \mathrm{H}, \mathrm{NH}) ;{ }^{13} \mathrm{C}$ NMR (DMSO-d6) $\delta 51.33,51.38,55.96,56.54,123.45,123.70$, $125.57,128.28,128.35,129.41,131.15,131.27$, 146.58, 146.68, 153.03, 157.56, 167.84; Anal. Calcd for $\mathrm{C}_{19} \mathrm{H}_{19} \mathrm{~N}_{5} \mathrm{O}_{3}: \mathrm{C}, 62.46 ; \mathrm{H}, 5.24 ; \mathrm{N}$, 19.17; found: C, 62.40; H, 5.30; N, 19.10 .

1-[2-Phenyl-5-(4-phenyl [1, 2, 3]-triazol-1ylmethyl) [1, 3, 4] oxadiazol-3-yl] ethanone (Va). Dirty white powder (60\%); decomposition $>300{ }^{\circ} \mathrm{C}$; IR (film), 3039 $(\mathrm{CH}$, aromatic), 2900 ( $\mathrm{CH}$, aliphatic), $1600(\mathrm{C}=\mathrm{N}) \mathrm{cm}-1$; ${ }^{1} \mathrm{H}$ NMR (DMSO-d6) $\delta 2.14\left(\mathrm{~s}, 3 \mathrm{H}, \mathrm{CH}_{3}\right), 5.57-5.60(\mathrm{~m}$, $\left.2 \mathrm{H}, \mathrm{CH}_{2}\right), 6.96(\mathrm{~s}, 1 \mathrm{H}$, oxadiazole $\mathrm{CH}), 7.30-7.34$ (m, $2 \mathrm{H}$, oxazolophenyl $\mathrm{H}-3,5), 7.36-7.44(\mathrm{~m}, 6 \mathrm{H}$, oxazolophenyl $\mathrm{H}-2,6,4$, phenyl $\mathrm{H}-3,4,5)$, 7.67.82( $\mathrm{m}, 2 \mathrm{H}$, phenyl $\mathrm{H}-6,2), 8.41(\mathrm{~s}, 1 \mathrm{H}$, triazole $\mathrm{H}$ ); ${ }^{13} \mathrm{C}$ NMR (DMSO-d6) $\delta$ 11.48, 51.26, 91.87, $122.87,123.19,125.45,126.91,127.12,128.36$, 129.15, 131.08, 136.30, 146.64, 157.96, 162.43; Anal. Calcd for $\mathrm{C}_{19} \mathrm{H}_{17} \mathrm{~N}_{5} \mathrm{O}_{2}$ : C, 65.69; $\mathrm{H}, 4.93 ; \mathrm{N}$, 20.16; found: C, 65.60; H, 4.90; N, 20.20. 
1-[2-(4-Nitrophenyl-5-(4-phenyl-[1, 2, 3]

triazol-1-ylmethyl)-[1, 3, 4]-oxadiazol-3-yl] ethanone (Vb). Dirty white powder $(60 \%)$; mp328-330 ${ }^{\circ} \mathrm{C}$; IR (film) $3070(\mathrm{CH}$, aromatic), $2900(\mathrm{CH}$, aliphatic), $1660(\mathrm{C}=\mathrm{O}), 1604(\mathrm{C}=\mathrm{N})$ $\mathrm{cm}-1$; $1 \mathrm{H}$ NMR (DMSO-d6) $\delta 1.92\left(\mathrm{~s}, 3 \mathrm{H}, \mathrm{CH}_{3}\right.$ ), 5.62-5.65 (m, 2H, $\left.\mathrm{CH}_{2}\right), 7.18(\mathrm{~s}, 1 \mathrm{H}$, oxadiazole $\mathrm{H})$, 7.32-7.34 (m, H, phenyl H-4), 7.42-7.45 (m, $2 \mathrm{H}$, phenyl $\mathrm{H}-3,5)$, 7.77-7.88( $\mathrm{m}, 4 \mathrm{H}$, phenyl $\mathrm{H}$ $6,2$, nitrophenyl $\mathrm{H}-2,6), 8.28(\mathrm{~d}, 2 \mathrm{H}, \mathrm{J}=8.8 \mathrm{~Hz}$, nitrophenyl $\mathrm{H}-3,5), 8.46(\mathrm{~s}, 1 \mathrm{H}$, triazole $\mathrm{H}) ; 13 \mathrm{C}$ NMR (DMSO-d6) $\delta 20.85,50.76,90.50,123.23$, 124.42, 125.59, 128.39, 129.20, 131.00, 142.01, 146.72, 148.85, 158.08, 164.90, 172.97. Anal. Calcd for $\mathrm{C}_{19} \mathrm{H}_{16} \mathrm{~N}_{6} \mathrm{O}_{4}: \mathrm{C}, 58.16 ; \mathrm{H}, 4.11 ; \mathrm{N}$, 21.42; found: C, 58.20; H, 4.10; N, 21.40 .

\section{1-[2-(3,4-Dimethoxyphenyl)-5-(4-phenyl-}

[1,2,3]triazol-1-ylmethyl)-[1,3,4]- oxadiazol-3yl-] -ethanone $(\mathrm{Vc})$. Dirty white powder $(60 \%)$; mp 278-280; IR (film) 3074(CH, aromatic), 2950 ( $\mathrm{CH}$, aliphatic), $1693(\mathrm{C}=0) \mathrm{cm}-1 ; 1 \mathrm{H}$ NMR (DMSO-d6) $\delta 2.04$ (s, 3H, CH $\mathrm{CH}_{3}, 3.62,3.63$ (s, $\left.6 \mathrm{H}, 2 \mathrm{OCH}_{3}\right), 5.10\left(\mathrm{~s}, 2 \mathrm{H}, \mathrm{CH}_{2}\right), 7.32-7.46(\mathrm{~m}$, $7 \mathrm{H}$, oxadiazole $\mathrm{H}$, dimethoxyphenyl $\mathrm{H}-2,5,6$, phenyl $\mathrm{H}-3,4,5)$, 7.81-7.83 $(\mathrm{m}, 2 \mathrm{H}$, phenyl $\mathrm{H}$ $6,2), 8.45(\mathrm{~s}, 1 \mathrm{H}$, triazole $\mathrm{H})$; Anal. Calcd for $\mathrm{C}_{21} \mathrm{H}_{21} \mathrm{~N}_{5} \mathrm{O}_{4}$ : C, 61.91; $\mathrm{H}, 5.20 ; \mathrm{N}, 17.19$; found: C, $61.80 ; \mathrm{H}, 5.30 ; \mathrm{N}, 17.20$.

\section{Antibacterial activity}

Results of the antibacterial and antifungal screening were verified as MICs; the MIC is the lowermost concentration of antibacterial /antifungal agents that causes almost complete inhibition of growth. The results are shown in Table 1. The inhibitory effects of the new compounds were screened against the following microorganisms (Klebsiella sp. (Kleb.), Listeria innocua (Lis.), Proteus vulgaris (Prot.), Micrococcus sp.(Micr.), Staphylococcus epidermidis (Staph.), Candida albicans (Ca. 1), Candida kruzi (Ca. 2), Klebsiella pneumonia (KI. Pn.), Escherichia coli (E. coli), Acinetobacter sp.(Acin.), Enterococcus faecium (Ent.), Vancomycin Resistant Staphylococcus aureus (VRSA), Bacillus sp. (Ba.), Streptococcus pyogenes (Strep.), Escherichia coli (E. coli 2), Enterococcus faecalis (Enter.), Sarcina lutea (Sar.), and Pseudomonas aeruginosa (Ps.). There were no activity except for compounds III, IVd and Vc which produced potent activity against Pseudomonas aeruginosa. Compounds III and IVc showed moderate activity against Staphylococcus epidermidis.

\section{Trypanosoma brucei bloodstream forms (BSF) in-vitro}

In this research, two strains of the bloodstream forms of Trypanosoma brucei were utilized. The first was a standard drug-sensitive laboratory strain of Trypanosoma brucei (s427-WT). The other was TbAT1-B48, which was derived from s427-WT through a genetic knockout of the TbAT1/P2 drug transporter, followed by exposure to a gradual increase in the concentration of pentamidine [19]. It is usually highly resistant to pentamidine and diminazene as well as melaminophenyl arsenicals, lacking both the TbAT1/P2 transporter and the high-affinity pentamidine transporter [18]. The results are shown in Table 2.

Table 1: Antibacterial effects of the new compounds (MIC, $\mu \mathrm{g} / \mathrm{ml}$ )

\begin{tabular}{lcc}
\hline Compound & $\begin{array}{c}\text { Staphylococcus } \\
\text { epidermidis }\end{array}$ & $\begin{array}{c}\text { Pseudomonas } \\
\text { aeruginosa }\end{array}$ \\
\hline II & $>100$ & $>100$ \\
III & 50 & $\mathbf{1 2 . 5}$ \\
IVb & $>100$ & $>100$ \\
IVc & 50 & $\mathbf{1 0 0}$ \\
IVd & $>100$ & $\mathbf{2 5}$ \\
Va & $>100$ & $>100$ \\
Vb & $>100$ & $>100$ \\
Vc & $>100$ & $\mathbf{5 0}$ \\
Ampicillin & 0.7 & $>\mathbf{1 0 0}$ \\
\hline
\end{tabular}

\section{Anti-kinetoplastid parasite activity}

\section{Promastigotes of Leishmania major and Leishmania mexicana}

The antileishmanial activity closely mirrored the activity against the related kinetoplastid parasite $T$. brucei (Table 2), with compounds II and IVb displaying $E_{50}$ values of $10.1 \pm 2.1$ $\mu \mathrm{M}$ and $18.0 \pm 0.8 \mu \mathrm{M}$, respectively, against $L$. major promastigotes.

Compounds II and IVb had moderate inhibitory effects against kinetoplastid parasite, while the other compounds showed no activity.

\section{Molecular docking results}

In an attempt to explore the mechanisms of action of the newly synthesized target compounds as anti-kinetoplastid parasite agents, the compounds were subjected to molecular docking studies using glyceraldehyde-3-phosphate dehydrogenase (GAPDH) which catalyzes the sixth reaction in the glycolytic sequence [20]) In kinetoplastids such as Trypanosoma and Leishmania, the glycolytic pathway is localized mainly in 
Table 2: Anti-kinetoplastid parasite effects of the new compounds $\left(\mathrm{EC}_{50}, \mu \mathrm{M}\right)$

\begin{tabular}{lcccc}
\hline Compound & $\begin{array}{c}\text { Trypanosoma brucei } \\
\text { brucei (WT) }\end{array}$ & $\begin{array}{c}\text { Trypanosoma brucei } \\
\text { brucei (B48) }\end{array}$ & $\begin{array}{c}\text { L. major, } \\
\text { promastigotes }\end{array}$ & $\begin{array}{c}\text { L. mexicana, } \\
\text { promastigotes }\end{array}$ \\
\hline II & $9.10 \pm 0.2$ & $10.7 \pm 0.7$ & $10.10 \pm 2.10$ & $5.10 \pm 0.3$ \\
III & $>100$ & $>100$ & $>100$ & $>100$ \\
IV a & $>100$ & $>100$ & $>100$ & $>100$ \\
IV b & $8.6 \pm 0.6$ & $8.8 \pm 0.60$ & $18 \pm 0.8$ & $28.10 \pm 3.80$ \\
IV c & $>100$ & $>100$ & $>100$ & $>100$ \\
IV d & $>100$ & $>100$ & $>100$ & $>100$ \\
Va & $>100$ & $>100$ & $>100$ & $>100$ \\
Vb & $>100$ & $>100$ & $>100$ & $>100$ \\
Vc & $>100$ & $>100$ & $>100$ & $>100$ \\
Pentamidine & $0.005 \pm 0.001$ & $0.37 \pm 0.01$ & $4.340 \pm 0.172$ & $1.247 \pm 0.113$ \\
\hline
\end{tabular}

Values are mean \pm SEM)

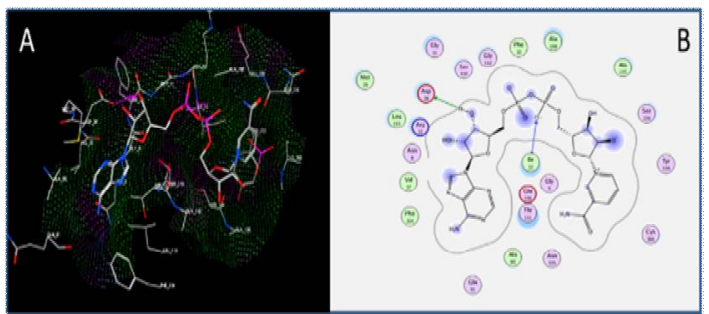

Figure 1: Binding of NAD inside glyceraldehyde-3phosphate dehydrogenase (GAPDH). (A) 3D interactions of NAD with (GAPDH) using MOE site finder program. The red dotted line represents $\mathrm{H}$ bonding interactions between $-\mathrm{OH}$ and $-\mathrm{PO}_{4}$ groups and Asp38 and lie13 amino acids. (B) 2D interactions of NAD with GAPDH

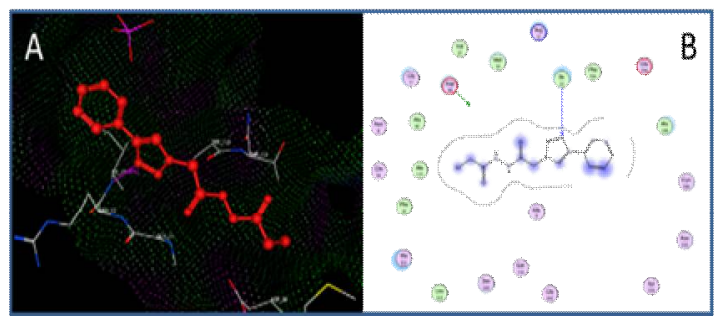

Figure 2: Binding of compound II inside GAPDH. (A) 3D interactions of compound II within GAPDH using MOE site finder program. The dotted lines represent three $\mathrm{H}$-bonding interactions between $\mathrm{N}$ of triazole moiety and Ile13. (B) 2D interactions of compound II with GAPDH.

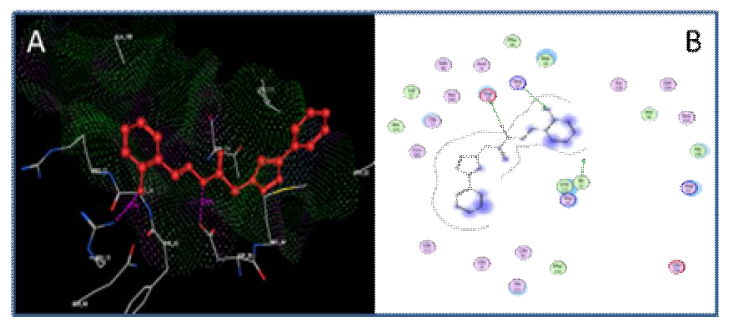

Figure 3: Binding of compound IVb inside GAPDH. (A) 3D interactions of compound IVb within GAPDH using MOE site finder program. The dotted lines represent two $\mathrm{H}$-bonding interactions between $\mathrm{NH}$ group and Asp38, and $\mathrm{OH}$ with Arg15. (B) 2D interactions of compound IVb with GAPDH
Table 3: Molecular modeling data for compounds II, $\mathrm{IVb}$ and NAD during docking in GAPDH

\begin{tabular}{|c|c|c|c|c|c|}
\hline $\begin{array}{l}\text { Compoun } \\
\text { d }\end{array}$ & $\begin{array}{c}\text { Affinit } \\
\text { y } \\
\text { (kcal/ } \\
\text { mol) }\end{array}$ & $\begin{array}{l}\text { No. of } \\
\text { Hydro } \\
\text { gen } \\
\text { bonds }\end{array}$ & \multicolumn{2}{|c|}{$\begin{array}{c}\text { Distance } \\
(\AA) \text { from } \\
\text { main } \\
\text { residue }\end{array}$} & $\begin{array}{c}\text { Functi } \\
\text { onal } \\
\text { group }\end{array}$ \\
\hline II & -16.34 & 1 & $\begin{array}{c}\text { Ile1 } \\
3\end{array}$ & $\begin{array}{l}2 . \\
41\end{array}$ & $\begin{array}{c}\mathrm{N} \text { of } \\
\text { triazole }\end{array}$ \\
\hline IV b & -16.22 & 2 & $\begin{array}{c}\text { Asp } \\
38 \\
\text { Arg } \\
15\end{array}$ & $\begin{array}{l}2 . \\
35 \\
2 . \\
67\end{array}$ & $\begin{array}{l}\mathrm{NH} \\
\mathrm{OH}\end{array}$ \\
\hline $\begin{array}{l}\text { Ligand } \\
\text { (NAD) }\end{array}$ & -15.24 & 2 & $\begin{array}{c}\text { Asp } \\
38 \\
\text { Ile1 } \\
3\end{array}$ & $\begin{array}{l}2 . \\
66 \\
2 . \\
52\end{array}$ & $\begin{array}{c}\mathrm{OH} \\
\mathrm{PO} 4\end{array}$ \\
\hline
\end{tabular}

glycosomes [23, 24]. The bindings of compounds II and IVb to GAPDH are shown in Figures 1 - 3.

\section{DISCUSSION}

The structure of chloroacetyl-triazole II was confirmed by NMR in which the new NMR peaks that appeared at $\delta 4.46$ (HNMR) and 44.47 (CNMR) were equivalent to $\mathrm{CH}_{2} \mathrm{Cl}$, and the presence of chloride in the mass spectra confirmed the structure of chloro-acetyl-triazole II. The disappearance of the carbonyl group peaks in the IR spectrum and $\mathrm{NH}$ peaks in the ${ }^{1} \mathrm{H}-\mathrm{NMR}$ spectra indicated the structure of oxadiazole-triazole III. The molecular structure of each azomethine derivative was confirmed by the appearance of new and characteristic de-shielded azomethine peaks $(\mathrm{CH}=\mathrm{N})$ and the disappearance of $\mathrm{NH}_{2}$ peaks. The azomethine $\mathrm{CH}$ peak appeared at $\delta 8.51-8.61 \mathrm{ppm}$, while the methoxy groups of azomethine derivatives appeared in the aliphatic region in the ${ }^{1} \mathrm{H}$-NMR or ${ }^{13} \mathrm{C}$-NMR spectra. The methoxy group peaks appeared at $\delta$ 3.37-3.85 ( ${ }^{1} \mathrm{H}-\mathrm{NMR}$ ) and 50-56 $\left({ }^{13} \mathrm{C}-\mathrm{NMR}\right) \mathrm{ppm}$. The new phenolic $(\mathrm{OH})$ peak of compound IVb appeared at $\delta 11.82 \mathrm{ppm}\left({ }^{1} \mathrm{H}\right.$ NMR). New NMR peaks (of methyl group) indicating the acetyl moiety appeared at $\delta 1.92-$ 
$2.14\left({ }^{1} \mathrm{H}-\mathrm{NMR}\right)$ and $11-20\left({ }^{13} \mathrm{C}-\mathrm{NMR}\right) \mathrm{ppm}$, and confirm the structure of oxadiazole derivatives Va-Vc.

Only compound oxadiazole III displayed activity against Pseudomonas aeruginosa, with an MIC of $12.5 \mu \mathrm{g} / \mathrm{mL}$, which may be attributed to the presence oxadiazole ring.

The chloroacetyl compound II and azomethine containing hydroxyl group IVb, displayed moderate activity against $T$. brucei, with $\mathrm{EC}_{50}$ values close to $10 \mu \mathrm{M}$, and importantly, no loss of activity against the multi-drug resistant strain B48. The moderate activities of compounds II and IVb may be due to their polarity.

It was observed that the $\mathrm{EC}_{50}$ values against $L$. mexicana were very similar to those found against $L$. major, confirming that the scaffolds have broad activity against kinetoplastid parasites, and that the anti-kinetoplastid activity could be further improved by exploring more of the structure-activity relationships and identifying the targets to enable structure-aided design.

Glyceraldehyde 3-phosphate dehydrogenase (GAPDH) is an attractive drug target in trypanosomes since their bloodstream forms lack the citric acid cycle and the classical electron transport chain in their minimalized mitochondrion, making them depend solely on glycolysis for their energy requirements. Glyceraldehyde 3-phosphate dehydrogenase from the pathogenic trypanosomatids $T$. brucei is quite similar to GAPDH from Leishmania Mexicana. However, the GAPDH in these pathogens is structurally different from the human GAPDH. This difference is exploited in the structure-based design of compounds that selectively inhibit the trypanosomatid enzymes but not the human homologue. Moreover, GAPDH was used in the present study as a target hunt. The novel synthesized triazoleoxazole scaffold has promising antimicrobial activity as a result of its multi-targets and mutual combination of two active structures.

\section{CONCLUSION}

The oxadiazole nucleus of compound Vc or nonacetylated III has important antibacterial activity, especially against Pseudomonas aeruginosa. However, the 2-hydroxy group in compound IVb and the chloroacetyl group in compound II strongly improve the anti-parasitic activities of these compounds; they may affect the conformation of the structure with respect to formation of $\mathrm{H}$-bonds with GAPDH receptors, if
GAPDH is confirmed to be the cellular target. Thus, the antimicrobial activity of the novel triazoles may be beneficial for the discovery of new antimicrobial drugs.

\section{DECLARATIONS}

\section{Acknowledgement}

The authors would like to thank Jouf University, $\mathrm{KSA}$, for funding this research through research Grand Project no. 378/37. In addition, the authors would like to thank Dr. Rania B Bakr for helping with molecular docking studies.

\section{Conflict of interest}

No conflict of interest is associated with this work.

\section{Contribution of authors}

We declare that this work was done by the authors named in this article and all liabilities pertaining to claims relating to the content of this article will be borne by the authors.

\section{Open Access}

This is an Open Access article that uses a funding model which does not charge readers or their institutions for access and distributed under the terms of the Creative Commons Attribution License (http://creativecommons.org/licenses/by/ 4.0) and the Budapest Open Access Initiative (http://www.budapestopenaccessinitiative.org/rea d), which permit unrestricted use, distribution, and reproduction in any medium, provided the original work is properly credited.

\section{REFERENCES}

1. Yao G, Haque S, Sha L, Kumaravel G, Wang J, Engber $T M$, Whalley ET, .Conlon PR, Chang H,.Kiesman WF, Petter RC. Synthesis of alkyne derivatives of a novel triazolopyrazine as $A 2 A$ adenosine receptor antagonists. Bioorg Med Chem Lett 2005; 15(3): 511515.

2. Sadana AK, Mirza Y, Aneja KR, Prakash O. Hypervalent iodine mediated synthesis of 1-aryl/hetryl-1, 2, 4-triazolo [4, 3-a] pyridines and 1-aryl/hetryl 5-methyl-1, 2, 4triazolo [4, 3-a] quinolines as antibacterial agents. Eur J Med Chem 2003; 38(5): 533-536.

3. Bussolari JC, Panzica RP. Synthesis and anti-HIV evaluation of 2', 3'-dideoxy imidazo-and v-triazolo [4, 5d] pyridazine nucleosides. Bioorg Med Chem 1999; 7(11): 2373-2379. 
4. Vu CB, Shields P, Peng B, Kumaravel G, Jin X, Phadke $D$, Wang J, Engber T, Ayyub E,.Petter RC. Triamino derivatives of triazolotriazine and triazolopyrimidine as adenosine $A 2 A$ receptor antagonists. Bioorg Med Chem Lett 2004; 14(19): 4835-4838.

5. Rodríguez $H$, Suárez $M$, Albericio $F$. Thiadiazines, $N, N$ heterocycles of biological relevance. Molecules. 2012; 17(7): 7612-7628.

6. Eweiss NF, Bahajaj AA, Elsherbini EA. Synthesis of heterocycles. Part VI. Synthesis and antimicrobial activity of some 4-amino-5-aryl-1, 2, 4-triazole-3-thiones and their derivatives. $J$ Heterocycl Chem 1986; 23(5): 1451-1458.

7. Sridhar SK, Saravanan M, Ramesh A. Synthesis and antibacterial screening of hydrazones, Schiff and Mannich bases of isatin derivatives. Eur J Med Chem 2001; 36(7): 615-625.

8. Gandini A, Prati F, Uliassi E, Bolognesi ML. Drug Discovery Strategies for the Generation of Multitarget Ligands against Neglected Tropical Diseases. Drug Sel An Evol Concept Med Chem 2017; 135-159.

9. Organization WH. Investing to overcome the global impact of neglected tropical diseases: third WHO report on neglected tropical diseases 2015. Vol. 3. World Health Organization; 2015.

10. Mondelaers A, Sanchez-Cañete MP, Hendrickx S, Eberhardt E, Garcia-H R, Lachaud L, Cotton J, Sanders $M$, Cuypers $B$, Imamura $H$, et al Genomic and molecular characterization of miltefosine resistance in Leishmania infantum strains with either natural or acquired resistance through experimental selection of intracellular amastigotes. PloS one. 2016 Apr 28; 11(4): e0154101.

11. Baker N, de Koning HP, Mäser P, Horn D. Drug resistance in African trypanosomiasis: the melarsoprol and pentamidine story. Trends Parasitol. 2013; 29(3): 110-118.

12. Campos MC, Leon LL, Taylor MC, Kelly JM. Benznidazole-resistance in Trypanosoma cruzi: evidence that distinct mechanisms can act in concert. Mol Biochem Parasitol. 2014; 193(1): 17-19.

13. Abdelgawad MA, Bakr RB, Omar HA. Design, synthesis and biological evaluation of some novel benzothiazole/benzoxazole and/or benzimidazole derivatives incorporating a pyrazole scaffold as antiproliferative agents. Bioorg Chem 2017; 74: 82-90.

14. Belal A, Abdelgawad MA. New benzothiazole/benzoxazole-pyrazole hybrids with potential as COX inhibitors: design, synthesis and anticancer activity evaluation. Res Chem Intermed. 2017; 43(7): 3859-3872.
15. Abdelgawad MA, Labib MB, Ali WA, Kamel G, Azouz AA, EL-Shaymaa EN. Design, synthesis, analgesic, antiinflammatory activity of novel pyrazolones possessing aminosulfonyl pharmacophore as inhibitors of COX-2/5LOX enzymes: Histopathological and docking studies. Bioorg Chem. 2018; 78: 103-114.

16. Youssif BGM. Synthesis and Biological Evaluation of Some New 1,2,3-Triazole Derivatives As Anti-microbial Agents. J Adv Chem. 2015; 11(2): 3473-3484.

17. Tarek N, Hassan HM, AbdelGhani SMM, Radwan IA, Hammouda O, El-Gendy AO. Comparative chemical and antimicrobial study of nine essential oils obtained from medicinal plants growing in Egypt. Beni-Suef Univ J Basic Appl Sci. 2014; 3(2): 149-156.

18. Bridges DJ, Gould MK, Nerima B, Mäser P, Burchmore RJ DKH. Loss of the high affinity pentamidine transporter is responsible for high levels of crossresistance between arsenical and diamidine drugs in African trypanosomes. Mol Pharmacol. 2007; 71: 10981108.

19. Matovu E, Stewart ML, Geiser F, Brun R, Mäser $P$, Wallace LJ, Burchmore RJ, Enyaru JC, Barrett MP, Kaminsky R ST. Mechanisms of arsenical and diamidine uptake and resistance in Trypanosoma brucei. Eukaryot Cell. 2003; 2(5): 1003-1008.

20. Munday JC, Eze AA, Baker N, Glover L, Clucas C, Aguinaga A D, Natto MJ, Teka IA, McDonald J, Lee RS et al Trypanosoma brucei aquaglyceroporin 2 is a highaffinity transporter for pentamidine and melaminophenyl arsenic drugs and the main genetic determinant of resistance to these drugs. J Antimicrob Chemother. 2013; 69(3): 651-663.

21. Al-Salabi MI, Wallace LJ, de Koning HP. A Leishmania major Nucleobase Transporter Responsible for Allopurinol Uptake is a Functional Homolog of theTrypanosoma brucei H2 Transporter. Mol Pharmacol. 2003; 63(4): 814-820.

22. Natto MJ, Savioli F, Quashie NB, Dardonville C, Rodenko $B$ de $K \mathrm{~K}$. Validation of novel fluorescence assays for the routine screening of drug susceptibilities of Trichomonas vaginalis. J Antimicrob Chemother. 2012; 67(4): 933943.

23. Wallace LJ, Candlish D, De Koning HP. Different substrate recognition motifs of human and trypanosome nucleobase transporters: selective uptake of purine antimetabolites. J Biol Chem. 2002; 277: 26149-26156.

24. Alzahrani KJ, Ali JA, Eze AA, Looi WL, Tagoe DN, Creek $D J$, Barrett MP, De Koning HP. Functional and genetic evidence that nucleoside transport is highly conserved in Leishmania species: Implications for pyrimidine-based chemotherapy. Int J Parasitol Drugs Drug Resist. 2017; 7(2): 206-226. 